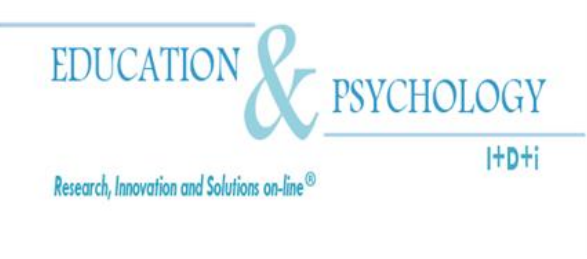

\title{
Autoconcepto y ansiedad social como variables predictoras del rendimiento académico de los adolescentes españoles con padres divorciados
}

\section{Mireia Orgilés ${ }^{1}$, Blair T. Johnson ${ }^{2}$, Tania B. Huedo- Medina $^{3}$, José P. Espada ${ }^{4}$}

\author{
${ }^{1}$ Departamento de Psicología de la Salud, \\ Universidad Miguel Hernández de Elche, Alicante, España \\ ${ }^{2}$ Department of Health Psychology, \\ University of Connecticut, Storrs, Estados Unidos \\ ${ }^{3}$ Department of Health Psychology, \\ University of Connecticut, Storrs, Estados Unidos \\ ${ }^{4}$ Departamento de Psicología de la Salud, \\ Universidad Miguel Hernández de Elche, Alicante, España
}

\section{España/EEUU}

Correspondencia: Mireia Orgilés. Universidad Miguel Hernández de Elche. Avda. de la Universidad s/n, Elche, 03202, Alicante. España. E-mail: morgiles@umh.es 


\section{Resumen}

Introducción. Estudios previos indican que el divorcio de los padres puede aumentar la vulnerabilidad de los hijos a desarrollar determinados problemas personales, como bajo rendimiento académico. El presente estudio examina el rendimiento académico de los adolescentes españoles con padres divorciados y su relación con el autoconcepto y la ansiedad social.

Método. La muestra está formada por 342 adolescentes con edades comprendidas entre 14 y 18 años, la mitad de ellos con padres divorciados, reclutados de 15 colegios ubicados en cinco provincias españolas. Los participantes informaron del número de sobresalientes y suspensos obtenidos y completaron el Cuestionario de Autoconcepto y la Escala de Ansiedad Social para Adolescentes.

Resultados. Los resultados indican que, en comparación con los adolescentes con padres divorciados, los que tenían padres casados (a) obtenían más suspensos, y (b) tenían un peor autoconcepto académico. El análisis de regresión múltiple reveló que los adolescentes con padres divorciados que tenían más probabilidad de obtener calificaciones de suspenso eran los varones, más mayores, con peor autoconcepto, y que vivían con la nueva pareja de la madre o padre.

Discusión. Se confirma que el divorcio de los padres se relaciona en los adolescentes españoles con peor rendimiento académico. Los hallazgos sugieren la importancia de detectar, desde el ámbito educativo, problemas escolares en los adolescentes cuyos padres se han divorciado con el objetivo de prevenir el fracaso escolar y otros problemas asociados.

Palabras Clave: Rendimiento académico, autoconcepto, ansiedad social, adolescentes españoles, padres divorciados. 


\title{
Self-concept and social anxiety as predictor variables of academic performance of Spanish adolescents with divorced parents
}

\begin{abstract}
Introduction. According to previous studies, when parents divorce it may increase the vulnerability of children to develop personal problems, such as lowering academic performance. This research examines the academic performance of Spanish children with divorced parents and its relation to academic self-concept and social anxiety.

Method. The sample includes 342 boys and girls aged between 14 and 18 years, half $(n=$ 171) with divorced parents, recruited from 15 schools in five counties of Spain. Participants provided information on the number of their courses evaluated as Excellent and Failure and completed the academic dimension of the Self-Description Questionnaire II and the Social Anxiety Scale for Adolescents.

Results. Compared to youth whose parents are living together, the results confirm that youth whose parents are divorced (a) failed more examinations, and (b) had a worse academic selfconcept. A multiple regression analysis revealed that it is more likely to drop for adolescent children of divorced parents who are male, older, have worse school self-concept, and live with a stepparent.

Discussion. Parental divorce is related to a worse academic performance in the Spanish adolescents. The findings suggest the importance of detecting, from the educational field, school problems in those adolescents whose parents have divorced to prevent the school failure and other associated problems.
\end{abstract}

Keywords: Academic performance, academic self-concept, social anxiety, Spanish adolescents, divorced parents

Received: $11 / 25 / 11$

Initial acceptance: $\quad 1223 / 12$

Final acceptance: 03/14/12 


\section{Introducción}

Desde que en 1981 se aprobó la ley del divorcio en España, el número de rupturas de pareja ha aumentado de forma considerable. España fue uno de los últimos países de la Unión Europea en permitir el divorcio, después de un largo período en el que no era posible disolver las uniones matrimoniales. La reforma de la ley en el año 2005 contribuyó a que el número de divorcios aumentara, debido a que a partir de ese momento no era necesaria la separación previa al divorcio, no se requerían unas causas determinadas para poder divorciarse, y el periodo mínimo necesario para solicitar el divorcio se redujo a tres meses desde la celebración del matrimonio. Junto con Bélgica y Hungría, España es uno de los países europeos con mayor número de divorcios; en concreto se producen dos rupturas por cada tres matrimonios que se celebran (Instituto de Política Familiar, 2010). El aumento de las rupturas y los nuevos modelos de funcionamiento familiar han generado la necesidad de llevar a cabo estudios que examinen cómo el divorcio afecta a los niños españoles para poder desarrollar estrategias y programas de apoyo. El divorcio representa un cambio en la estructura y el funcionamiento de la familia que puede afectar de forma significativa a los niños (Sandler, Tein, Mehta, Wolchik, y Ayers, 2000). En investigaciones con muestras españolas las rupturas de pareja se han relacionado con diversos problemas en los hijos, como el trastorno de ansiedad por separación (Orgilés, Espada, y Méndez, 2007), los miedos escolares (Orgilés, Espada, Méndez, y GarcíaFernández, 2007) o los problemas de conducta (Cantón, Cortés, y Justicia, 2002). Diversos estudios confirman que los niños con padres divorciados tienen un peor rendimiento académico (Martínez y Forgatch, 2002; Potter, 2010; Tillman, 2007), sin embargo la evidencia científica procede principalmente de investigaciones llevadas a cabo en Estados Unidos. Estudios previos sugieren que existen determinadas variables que pueden moderar el efecto del divorcio, como el grado de conflicto entre los padres (Ghazarian y Buehler, 2010) o vivir con la nueva pareja de la madre o padre (Klomegah, 2007). Potter (2010) sugiere que es el descenso en el bienestar psicosocial de los niños después del divorcio de los padres lo que ayuda a explicar su relación con el bajo rendimiento académico.

En población general el rendimiento académico se ha asociado a menudo con el autoconcepto académico y la ansiedad social. Marsh y Shavelson (1985) definen el autoconcepto como la percepción que una persona tiene sobre sí mismo. El autoconcepto se desarrolla durante la adolescencia y depende, entre otros factores, del entorno familiar. El autoconcepto académico se ha considerado como predictor del rendimiento académico en los adolescentes 
(Boulter, 2002; Guay, Larose, y Boivin, 2004), y puede considerarse un factor de peso al explicar el éxito o fracaso escolar (Marsh y Yeung, 1997). Algunos estudios indican que comparados con los niños cuyos padres permanecen juntos, aquellos con padres divorciados tienen puntuaciones más bajas en autoconcepto (e.g., Sweeney y Bracken, 2000; Evans, Kelley, y Wanner, 2001). Otro factor que puede influir en los resultados académicos, pero que todavía no se ha estudiado en los niños con padres divorciados, es la ansiedad social. Se trata de uno de los problemas más frecuentes en los adolescentes y se puede definir como el miedo intenso a situaciones sociales y el intento frecuente de evitarlas. Conforme los adolescentes son más autónomos, las relaciones con los iguales y con el sexo opuesto cobran más importancia, especialmente la competencia social y el miedo a la evaluación negativa (Inglés, 2003). Estudios previos con adolescentes de la población general han mostrado que la ansiedad social implica una menor participación en clase, y que facilita el abandono escolar o un menor rendimiento académico (Francis y Radka, 1995). La necesidad de obtener buenos resultados académicos es también una fuente de estrés para los adolescentes, que a menudo expresan ansiedad ante situaciones de evaluación (Onyeizugbo, 2010). Los adolescentes que tienen más relaciones con sus iguales suelen manifestar niveles más altos de bienestar psicológico (Chow, 2010). El éxito en las relaciones interpersonales se relaciona positivamente con la adaptación escolar y el rendimiento académico, mientras que las dificultades en las relaciones interpersonales y la ansiedad social se asocian a un menor rendimiento académico (Inglés, 2003). La relación entre el rendimiento académico y la ansiedad social no es tan clara en los niños con padres divorciados, ya que no se han llevado a cabo estudios con población adolescente.

\section{Objetivos}

La frecuencia de los divorcios en España y las implicaciones del abandono escolar, junto con la falta de estudios con población española que examinen directamente dichas variables, justifican la necesidad de llevar a cabo este estudio. El objetivo es analizar cómo los resultados académicos se relacionan con el autoconcepto y la ansiedad social en los adolescentes con padres divorciados. En primer lugar, se examinan las diferencias en el rendimiento académico, el autoconcepto y la ansiedad social en función de tener padres divorciados o casados. En segundo lugar, se analiza la relación entre el rendimiento académico y la ansiedad social, el autoconcepto y algunas variables individuales y familiares en los adolescentes con padres divorciados. Además, se examina qué variables predicen el número de suspensos. 


\section{Método}

\section{Participantes}

Para reclutar la muestra, se contactó con 15 colegios de cinco provincias españolas. Tres colegios se seleccionaron del norte de España, tres de la parte central, dos del este, cuatro del suroeste y tres del sur. De los 15 colegios, nueve eran de ciudades costeras y el resto del interior. Se incluyeron colegios ubicados en zonas rurales y urbanas. Los directores de los colegios y los padres ofrecieron su consentimiento para el estudio. Los adolescentes cuyos padres firmaron el consentimiento informado (aproximadamente el 99\% del total) completaron los cuestionarios. El estudio recibió la aprobación del Comité de Ética de la institución que lo llevó a cabo.

De los 1,216 adolescentes, entre 14 y 18 años, que completaron las pruebas, el 20\% tenían padres divorciados. Se excluyeron a los adolescentes que vivían en una familia monoparental por alguna causa diferente al divorcio de los padres, como ser huérfano o no haber conocido nunca a su madre o padre. La muestra final estaba formada por 171 participantes con padres divorciados (14\% del total). Se seleccionó un grupo de comparación con las mismas características de edad, sexo, nivel socioeconómico y ubicación geográfica pero con padres casados. Todos los niños que participaron en el estudio eran españoles.

El grupo de adolescentes con padres divorciados tenía una edad media de 15.80 años $(D T=0.76)$, el $42 \%$ eran chicos $(n=72)$ y el $58 \%$ chicas $(n=99)$. La edad media en el momento del divorcio de los padres era de 7.81 años $(D T=4.22)$, en un rango de 0 a 17 años. En la mayoría de los casos la madre tenía la custodia ( $87 \%$ vs. $6 \%$ de padres); la custodia estaba compartida en el $5 \%$ de los casos, y en el $2 \%$ de los casos otros miembros de la familia, normalmente los abuelos, tenían la custodia de los adolescentes. Del grupo con padres divorciados, el $15 \%$ vivían con la madre o el padre y su nueva pareja. Además, el 44\% tenían hermanos. La relación entre los padres divorciados fue percibida por los adolescentes como buena, es decir sin conflicto, por el $72 \%$ de los adolescentes; el 19\% la consideraban inexistente (informaban de que sus padres no tenían relación) y el $9 \%$ la definían como conflictiva. El grupo de participantes con padres casados estaba formado por 171 adolescentes con una edad media de 15.91 años $(D T=0.77)$, de los que el $37 \%$ eran chicos $(n=64)$ y el $63 \%$ chicas $(n=107)$. 


\section{Procedimiento}

Los participantes completaron los cuestionarios en las aulas, en grupos de 20 o 30 estudiantes, siendo contrabalanceado el orden de las pruebas. Las instrucciones escritas se leyeron en voz alta y se resolvieron las dudas. Se proporcionó un sobre a cada participante para que metieran dentro los cuestionarios una vez completados, para asegurar el anonimato de las respuestas.

\section{Instrumentos}

La información referente a variables demográficas y familiares se recogió a través de cuestionarios. Para examinar el rendimiento académico se solicitó a los participantes que indicaran el número de asignaturas en las que habían obtenido la calificación de sobresaliente y de suspenso. Obtener una calificación de suspenso implica que el estudiante no ha alcanzado los objetivos de su nivel, con una puntuación menor a un 5. La puntuación más alta, sobresaliente, significa haber obtenido 9 o más puntos en la escala e indica una ejecución brillante por parte del estudiante. El presente estudió empleó el número de sobresalientes y el número de suspensos como variables dependientes por considerar que son las medidas más informativas del rendimiento académico (e.g., Inglés, 2009).

El autoconcepto académico se evaluó mediante el Cuestionario de Autoconcepto, versión breve (SDQ; Marsh, 1989; Marsh, Relich \& Smith, 1983). Se utilizaron únicamente las subescalas de autoconcepto en matemáticas, verbal y escolar. La versión española del SDQ se desarrolló mediante el método de traducción-retrotraducción. Un nativo inglés tradujo los ítems de inglés a español y de nuevo de español a inglés. Se compararon ambas versiones en inglés para examinar si existían diferencias entre ellas. El cuestionario mantiene sus buenas propiedades psicométricas en la muestra española, con una buena fiabilidad, $\alpha=.82$, apropiada validez factorial y adecuada validez convergente y discriminante (e.g., media $r=.35$ para las dimensiones académicas, y media $r=.05$ en las escalas que miden ansiedad y búsqueda de sensaciones).

La Escala de Ansiedad Social (SAS-A; La Greca y Stone, 1993) en su adaptación para adolescentes españoles (La Greca y López, 1998) se aplicó para evaluar la ansiedad social. El instrumento tiene 22 ítems, de los que 18 son ítems referentes a uno mismo (por ejemplo, "Siento que los demás se rien de mî") y el resto son ítems neutros (por ejemplo, "Me gusta hacer deporte"). Las opciones de respuesta siguen una escala tipo likert de cinco puntos, con 
un rango de 1 (nada verdadero) a 5 (siempre verdadero). La SAS-A incluye tres subescalas: Miedo a la evaluación negativa (FNE), Evitación social y estrés específico en situaciones nuevas o con personas desconocidas (SAD-N), y Evitación social y estrés con los iguales (SAD-G). Las puntuaciones podían oscilar de 1 a 90 para la puntuación total, de 1 a 40 para la subescala FNE, $\alpha=.84$, de 1 a 30 para la subescala SAD-N, $\alpha=.79$, y de 1 a 20 para la subescala SAD-G, $\alpha=.72$. La fiabilidad era buena, $\alpha=.84$ para la muestra total y $\alpha=.86$ para la submuestra de adolescentes con padres divorciados.

\section{Análisis de datos}

Las diferencias entre los adolescentes con padres divorciados y padres casados se examinaron mediante pruebas $t$ de student para la comparación entre dos muestras independientes. La relación entre ansiedad social, autoconcepto y rendimiento académico en los participantes con padres divorciados se evaluó mediante análisis de regresión univariados. En un análisis se utilizó como variable dependiente el número de asignaturas evaluadas como sobresalientes y en el otro el número de asignaturas evaluadas como suspensos. Finalmente se llevó a cabo un análisis de regresión múltiple incluyendo todas las variables para controlar las variables moderadores y sus interacciones tratando de hallar el mejor modelo que explicara el número de suspensos en los adolescentes con padres divorciados.

\section{Resultados}

\section{Diferencias entre adolescentes con padres divorciados y casados}

Los adolescentes cuyos padres estaban divorciados obtuvieron más asignaturas evaluadas como suspensos que aquéllos cuyos padres estaban casados $(p<.05, t=-2.44, d=$ 0.27). También manifestaron peor autoconcepto en la subescala escolar $(p<.05, t=-2.06, d=$ -0.22) (Tabla 1). No se hallaron diferencias significativas entre los dos grupos en la puntuación total de la Escala de Ansiedad Social ni en ninguna de sus subescalas. La región y el colegio de los participantes no afectaron a los resultados, de modo que no se hallaron diferencias entre los colegios de zonas rurales y urbanas o en relación a la situación geográfica de los participantes (norte, centro, este, sudeste y sur de España). 
Tabla 1. Diferencias en Rendimiento Académico, Autoconcepto Académico y Ansiedad Social entre los Adolescentes con Padres Divorciados y no Divorciados $(N=342)$

\begin{tabular}{|c|c|c|c|c|c|c|c|c|}
\hline & \multicolumn{4}{|c|}{ Estado civil de los padres } & \multirow[b]{3}{*}{$t$} & \multirow[b]{3}{*}{$p$} & \multirow[b]{3}{*}{$d$} \\
\hline & & \multicolumn{2}{|c|}{$\begin{array}{l}\text { Divorciados } \\
(\mathrm{n}=171)\end{array}$} & \multicolumn{2}{|c|}{$\begin{array}{l}\text { Casados } \\
(\mathrm{n}=171)\end{array}$} & & & \\
\hline & & $M$ & $D T$ & $M$ & $D T$ & & & \\
\hline \multirow{2}{*}{$\begin{array}{l}\text { Rendimiento } \\
\text { académico }\end{array}$} & $\begin{array}{l}\text { Número de } \\
\text { suspensos }\end{array}$ & 1.67 & $(2.10)$ & 1.13 & $(1.92)$ & -2.44 & .015 & 0.27 \\
\hline & $\begin{array}{l}\text { Número de } \\
\text { sobresalientes }\end{array}$ & 1.26 & $(1.73)$ & 1.59 & $(2.30)$ & 1.51 & .13 & -0.16 \\
\hline \multirow{3}{*}{$\begin{array}{l}\text { Autoconcepto } \\
\text { académico }\end{array}$} & Escolar & 11.67 & $(4.07)$ & 12.57 & $(4.02)$ & -2.059 & .040 & -0.22 \\
\hline & Matemático & 12.43 & $(6.52)$ & 13.04 & $(5.57)$ & -0.93 & .35 & -0.100 \\
\hline & Verbal & 17.49 & $(6.54)$ & 17.97 & $(6.65)$ & -0.67 & .50 & -0.072 \\
\hline \multirow{4}{*}{$\begin{array}{l}\text { Ansiedad } \\
\text { Social }\end{array}$} & FNE & 19.20 & $(5.97)$ & 18.88 & $(6.11)$ & -0.49 & .62 & -0.053 \\
\hline & SAD-N & 14.27 & $(4.25)$ & 14.77 & $(4.74)$ & 1.021 & .31 & 0.11 \\
\hline & SAD-G & 7.19 & $(2.62)$ & 7.60 & $(3.03)$ & 1.35 & .18 & 0.14 \\
\hline & Puntuación total & 40.66 & (11.03) & 41.25 & (12.27) & 0.47 & .64 & 0.051 \\
\hline
\end{tabular}

FNE = Miedo a la evaluación negativa; SAD-N = Evitación social y estrés en nuevas situaciones; SAD-G = Evitación social y estrés en general; $d=$ Tamaño del efecto (corregido por el tamaño de la muestra).

\section{Divorcio y Rendimiento Académico}

El rendimiento académico de los adolescentes con padres divorciados se examinó en función de la edad, sexo, autoconcepto académico, ansiedad social, tiempo transcurrido desde la ruptura de los padres, la convivencia con la nueva pareja de la madre o padre, y la calidad de la relación entre los padres percibida por los hijos (ver Tabla 2). Los análisis en función de la edad muestran que cuanto más mayor es el adolescente mayor es el número de suspensos. Se hallaron diferencias significativas en el número de suspensos en función del sexo, de forma que los chicos tenían más calificaciones de suspenso $(M=2.31 ; D T=2.49)$ que las chicas $(M$ $=1.28 ; D T=1.73)$. No se hallaron diferencias en el número de sobresalientes en función de la edad o del sexo. Los resultados revelan que el autoconcepto se relaciona con el rendimiento académico. A peor autoconcepto más número de suspensos, y a mejor autoconcepto más sobresalientes. La ansiedad social no influye ni en el número de suspensos ni en el número de sobresalientes $(p>.05)$.

El tiempo transcurrido desde la separación de los padres no se relacionó con el rendimiento académico $(p>.05)$. Tampoco se halló asociación con la relación entre los padres percibida por los hijos, tener hermanos o vivir con la nueva pareja de la madre o padre. 
Tabla 2. Rendimiento académico en función de las características de los adolescentes cuyos padres están divorciados $(N=171)$

\begin{tabular}{|c|c|c|c|c|c|}
\hline & & \multicolumn{4}{|c|}{ Rendimiento académico } \\
\hline \multirow{2}{*}{\multicolumn{2}{|c|}{ Variables moderadoras }} & \multicolumn{2}{|c|}{ Número de suspensos } & \multicolumn{2}{|c|}{ Número de sobresalientes } \\
\hline & & $\beta^{\mathrm{a}}$ & $p$ & $\beta^{\mathrm{a}}$ & $p$ \\
\hline \multicolumn{2}{|l|}{ Edad } & .23 & .002 & -.11 & .16 \\
\hline \multicolumn{2}{|l|}{$\begin{array}{l}\text { Sexo } \\
\text { femenino }^{b}\end{array}$} & -.23 & .002 & .14 & .075 \\
\hline \multirow{3}{*}{$\begin{array}{l}\text { Autoconceptc } \\
\text { académico }\end{array}$} & Escolar & -.42 & .000 & .44 & .000 \\
\hline & Matemático & -.25 & .001 & .28 & .000 \\
\hline & Verbal & -.25 & .001 & .35 & .000 \\
\hline \multirow{4}{*}{$\begin{array}{l}\text { Ansiedad } \\
\text { social }\end{array}$} & FNE & -.11 & .17 & .028 & .71 \\
\hline & SAD-N & -.10 & .203 & .043 & .58 \\
\hline & SAD-G & .049 & .527 & -.069 & .37 \\
\hline & Puntuación total & -.084 & .277 & .015 & .84 \\
\hline \multirow{4}{*}{$\begin{array}{l}\text { Variables } \\
\text { relacionadas } \\
\text { con el } \\
\text { divorcio de } \\
\text { los padres }\end{array}$} & $\begin{array}{l}\text { Tiempo desde el } \\
\text { divorcio }\end{array}$ & .092 & .23 & -.013 & .86 \\
\hline & $\begin{array}{l}\text { Calidad de la } \\
\text { relación entre los } \\
\text { padres }\end{array}$ & .025 & .75 & .022 & .77 \\
\hline & $\begin{array}{l}\text { Vivir con la } \\
\text { nueva pareja de } \\
\text { la madre o padre }\end{array}$ & -.15 & .06 & .010 & .93 \\
\hline & Tener hermanos & -.041 & .60 & .087 & .26 \\
\hline
\end{tabular}

FNE = Miedo a la evaluación negativa; SAD-N = Evitación social y estrés en nuevas situaciones; SAD-G = Evitación social y estrés en general; ${ }^{a}$ Valores positivos indican mejor rendimiento. ${ }^{\mathrm{b}} \mathrm{Chico}$ codificado como 0 , chica codificado como 1 ; Tener hermanastros codificado como 0 , no tener codificado como 1.

\section{Predicción del número de suspensos}

Se llevó a cabo un análisis de regresión múltiple para explicar las variables que afectan al número de suspensos en los adolescentes con padres divorciados. Los mejores predictores fueron el sexo, la edad, vivir con la nueva pareja de la madre o padre, y el autoconcepto escolar, con una explicación del $28 \%$ de la varianza ( $\mathrm{R}^{2}$ ajustada). De acuerdo al modelo obtenido, el número de suspensos es mayor en los chicos, de más edad, con peor autoconcepto, que viven con la nueva pareja de la madre o padre.

Tabla 3. Predictores del número de suspensos en los adolescentes con padres divorciados $(N=171)$ 


\begin{tabular}{|l|c|c|}
\hline \multirow{2}{*}{ Predictores } & \multicolumn{2}{|c|}{ Número de suspensos } \\
\cline { 2 - 3 } & $\beta$ & $p$ \\
\hline $\begin{array}{l}\text { Sexo } \\
\text { masculino }\end{array}$ & -.22 & .003 \\
\hline Edad & .19 & .009 \\
\hline Autoconcepto & -.40 & .001 \\
\hline $\begin{array}{l}\text { Vivir con la nueva pareja de la } \\
\text { madre o padre }\end{array}$ & -.77 & .007 \\
\hline $\begin{array}{l}\text { a } \\
\text { vivico codificado como } 0 \text {, chica codificado como } 1 \text {; }^{\mathrm{b}} \text { Vivir con la nueva pareja de la madre o padre codificado como 0, no }\end{array}$ \\
\hline
\end{tabular}

\section{Discusión y conclusiones}

El presente estudio examina las variables que se relacionan con el rendimiento académico en los adolescentes con padres divorciados. En primer lugar se evalúan las diferencias en función de la estructura familiar. No se hallaron diferencias significativas en el número de sobresalientes, pero de acuerdo a lo esperado los adolescentes con padres divorciados obtienen más suspensos que los que tienen padres casados. Este hallazgo se relaciona con resultados de estudios previos que indican que los adolescentes con padres divorciados tienen peor rendimiento académico que aquéllos cuyos padres viven juntos (e.g. Tillman, 2007). Los adolescentes con padres divorciados tienen también peor autoconcepto académico, de modo que se perciben menos competentes en situaciones relacionadas con este área. Los resultados son consistentes con la investigación previa que muestra que el autoconcepto en los adolescentes con padres divorciados es peor que el de aquéllos que proceden de familias con padres casados (e.g., Sweeney y Bracken, 2000). Sin embargo, en contra de lo esperado, no se hallaron diferencias en función de la ansiedad social. Desafortunadamente no se han encontrado estudios previos que relacionen la ansiedad social con el divorcio de los padres, por lo que los resultados de nuestro estudio no se han podido comparar con la evidencia previa. Después del divorcio de los padres, los niños deben afrontar nuevas situaciones sociales derivadas de los cambios que se producen al romper la pareja. A menudo se produce un cambio de casa, colegio, vecindario y en ocasiones incluso de ciudad, lo que implica hacer frente a nuevas situaciones sociales con amigos, compañeros de clase, vecinos, y amigos de los padres, entre otros. Aunque esta cuestión no está clara, la exposición de los adolescentes con padres divorciados a una mayor variedad de situaciones sociales podría generar en ellos menos ansiedad a interactuar socialmente, por lo que a pesar de lo esperado sus puntuaciones en ansiedad social no difieren de las del grupo con padres casados. 
Uno de los objetivos principales de este estudio era explicar el rendimiento académico en los adolescentes con padres divorciados. Los resultados del análisis de regresión múltiple indican que los mejores predictores del número de suspensos son el sexo, la edad, vivir con la nueva pareja de la madre o padre, y el autoconcepto escolar. Los chicos obtuvieron más suspensos que las chicas, de acuerdo a los estudios internacionales que indican que el rendimiento académico es mejor en las chicas que en los chicos con padres divorciados y que los chicos suelen tener más problemas académicos después del divorcio de los padres (Elder y Russell, 1996; Kaye, 1989). Es posible, como sugiere Nielsen (1999), que los chicos estén más expuestos a los efectos del divorcio de los padres que las chicas, porque según los estudios de este autor las madres tienden a realizar comentarios más negativos sobre la esposa actual de los padres que éstos; Nielsen (1999) mantiene que las madres divorciadas suelen ver a sus hijos como confidentes lo que podría interferir en la relación con los padres. Cuando el padre y el niño no mantienen un contacto frecuente después del divorcio, Nielsen predice más problemas psicológicos, académicos, sociales, sexuales y emocionales en los niños que en las niñas. Los resultados de nuestro estudio también indican que los adolescentes de más edad obtenían más suspensos que los más jóvenes. Aunque la razón no está clara, se sugiere que el nivel de demanda en los cursos avanzados es mayor, de modo que parece lógico que los adolescentes de más edad obtengan peores resultados. Consistente con estudios previos en la población general (Boulter, 2002; Guay et al., 2004) el número de suspensos se relaciona también con el autoconcepto académico. Como se esperaba, la relación entre autoconcepto académico y rendimiento académico hallado en numerosos estudios con población general ocurre también en los adolescentes con padres divorciados. Nuestros resultados muestran que los que obtienen más supensos viven con la nueva pareja de la madre o del padre, de acuerdo a estudios previos. El metaanálisis de Amato y Keith (1991) revela que los niños que viven con las nuevas parejas de los padres muestran un peor rendimiento académico, en comparación con los que viven con ambos padres. Amato y Keith mantienen que la relación de los padres con sus nuevas parejas puede ser estresante para los niños porque suele implicar un cambio de hogar a un nuevo vecindario o ciudad, y la necesidad de adaptarse a nuevas rutinas y normas. Las relaciones con las nuevas parejas es una fuente de estrés para los niños (Amato y Sobolewski, 2001) y en respuesta a ello, algunos menores, especialmente los adolescentes, pueden reaccionar con hostilidad y rechazo. Finalmente, mientras que la ansiedad social se ha relacionado con el rendimiento académico bajo en la población general (e.g., Van Amerigen, Mancini y Farvolden, 2003), dicha asociación no se ha hallado en nuestra muestra de 
adolescentes con padres divorciados. La falta de estudios previos sobre este tema en población adolescente con padres divorciados dificulta la comparación de estos resultados.

El estudio presenta algunas limitaciones. La principal es que se trata de un estudio transversal y no longitudinal. Además, debido al tamaño de la muestra no pudo llevarse a cabo un modelo explicatorio completo del rendimiento académico y la estructura familiar. Estudios futuros con muestras mayores son necesarios para lograr ese objetivo. El estudio tiene también algunas fortalezas e importantes conclusiones. En primer lugar, se confirma por primera vez con una muestra española que los adolescentes con padres divorciados tienen peor rendimiento académico que los que viven con ambos padres, y que su autoconcepto académico es también peor, probablemente como resultado de sus peores resultados académicos. Con relación a la recencia de la ley del divorcio en España, aprobado en 1981, y su aceptación social, los resultados de nuestro estudio indican que los adolescentes con padres divorciados tienen experiencias similares a los adolescentes de otros países en los que el divorcio es menos reciente. El intento de examinar la relación entre el divorcio de los padres y el rendimiento académico, autoconcepto y ansiedad sólo reveló pocas diferencias significativas con tamaños del efecto pequeños, sugiriendo que los adolescentes con padres divorciados y casados son más similares que diferentes en dichas variables. En segundo lugar, por primera vez se examina el autoconcepto y la ansiedad social como variables predictoras de los suspensos en los adolescentes con padres divorciados. Las implicaciones a nivel educativo de los hallazgos son claras. A pesar de las pocas diferencias encontradas en algunas variables, los adolescentes con padres divorciados obtienen más suspensos que los que viven con ambos padres, lo que implica que su rendimiento académico es peor. Se sugiere la importancia de detectar lo antes posible, desde el ámbito educativo, problemas escolares en los adolescentes con padres divorciados para prevenir el fracaso escolar y otros problemas asociados como el bajo autoconcepto. 


\section{Referencias}

Amato, P. R., y Keith, B. (1991). Parental divorce and the well-being of children: A metaanalysis. Psychological Bulletin. 110 (1), 26-46.

Amato, P. R., y Sobolewski, J. M. (2001). The effects of divorce and marital discord on adult children's psychological well-being. American Sociological Review, 66(6), 900-921.

Boulter, L. T. (2002). Self-concept as a predictor of collage freshman academic adjustment. College Student Journal, 36(2), 234-246.

Brown, N. W., y Cross, E. J. (1997). Coping resources and family environment for engineering students. College Student Journal, 31(2), 282-288.

Cantón, J., Cortés, M. R., y Justicia, M. D. (2002). Las consecuencias del divorcio en los hijos. Psicopatología Clínica, Legal y Forense, 2(3), 47-66.

Chow, H. P. H. (2010). Predicting academic success and psychological wellness in a sample of Canadian undergrate students. Electronic Journal of Research in Educational Psychology, 8(2), 473-496.

Elder, G. H., y Russell, S. T. (1996). Academic performance and future aspirations. En R. L. Simons \& Associates (Eds. ). Understanding differences between divorced and intact families: stress, interaction and child outcome (pp. 172-192). Thousand Oaks, CA: Sage.

Evans, M. D. R., Kelley, J. y Wanner, R. (2001). Educational attainment of children of divorce: Australia, 1940-90. Journal of Sociology, 37, 275-297.

Francis, G., y Radka, D. F. (1995). Social anxiety in children and adolescents. En M. B. Stein (Ed.). Social phobia: Clinical and research perspectives (pp. 119-143). New York: Guildford Press.

Ghazarian, S. R. y Buehler, C. (2010). Interparental conflict and academic achievement: an examination of mediating and moderating factors. Journal of Youth Adolescence. doi: 10.1007/s10964-008-9360-1

Guay, F., Larose, S., y Boivin, M. (2004). Academic self-concept and educational attainment level: a ten-year longitudinal study. Self and identity, 3(1), 53-68.

Hannum, J. W., y Dvorak, D. M. (2004). Effects of family conflict, divorce, and attachment patterns on the psychological distress and social adjustment of college freshmen. Journal of College Student Development, 45(1), 27-43.

Inglés, C. J. (2003). Programa PEHIA. Enseñanza de habilidades interpersonales para adolescentes. Madrid: Pirámide. 
Inglés, C. J. (2009). Conducta prosocial y rendimiento académico en estudiantes españoles de Educación Secundaría Obligatoria. Anales de Psicologia, 25, 93-101.

Institute for Families Policies (2010). Informe de la evolución de la familia en Europa. http://www. ipfe. org/Informe_Evolucion_Familia_Europa_2009_def_esp.pdf.

Kaye, S. H. (1989). The impact of divorce on children's academic performance. Journal of Divorce, 3, 283-298.

Klomegah, R. Y. (2007). Predictors of academic performance of university students: an application of the goal efficacy model. College Student Journal, 41, 407-415.

La Greca, A. M., y López, N. (1998). Social anxiety among adolescents: Linkages with peer relations and friendships. Journal of Abnormal Child Psychology, 26, 83-94.

La Greca, A. M., y Stone, W. L. (1993). The Social Anxiety Scale for Children-Revised: Factor structure and concurrent validity. Journal of Clinical Child Psychology, 22, 17-27.

McLanahan, S. S., y Sandefur, G. (1994). Growing up with a single parent: What hurts, what helps. Cambridge, MA: Harvard University Press.

Marsh, H. W. (1989). Age and sex effects in multiple dimensions of self-concept: Preadolescence to early adulthood. Journal of Educational Psychology, 81, 417-430.

Marsh, H. W., Relich, J. D., y Smith, I. D. (1983). Self-concept: The construct validity of interpretations based upon the SDQ. Journal of Personality and Social Psychology, 45, 173-187.

Marsh, H. W., y Yeung, A. S. (1997). Causal effects of academic self-concept on academic achievement: Structural equation models of longitudinal data. Journal of Educational Psychology, 89, 41-54.

Martinez, C. R., \& Forgatch, M. S. (2002). Adjusting to change: Linking family structure transitions with parenting and boys' adjustment. Journal of Family Psychology, 16, 107-117.

Marsh, H. W. y Shavelson, R. (1985). Self-concept: Its multifaceted, hierarchical structure. Educational Psychologist, 20, 107-125.

Nielsen, L. (1999). College aged students of divorced parents: Facts and fiction. College Student Journal, 33(4), 543-572.

Olivares, J., Ruiz, J., Hidalgo, M. D., García-López, L. J., Rosa, A. I., y Piqueras, J. A. (2005). Social Anxiety Scale for Adolescents, SAS-A. Psychometric properties in a Spanish speaking population. International Journal of Clinical and Health Psychology, 5(1), 85-97. 
Onyeizugbo, E. U. (2010). Self-efficacy, gender and trait anxiety as moderators of test anxiety. Electronic Journal of Research in Educational Psychology, 8(1), 299-312.

Orgilés, M., Espada, J. P., y Méndez, X. (2007). Trastorno de ansiedad por separación en hijos de padres divorciados. Psicothema, 20(3), 383-388.

Orgilés, M., Espada, J. P., Méndez, X., y García-Fernández, J. M. (2007). Miedos escolares en hijos de padres divorciados y no divorciados. International Journal of Clinical and Health Psychology, 8(3), 693-703.

Potter, D. (2010). Psychosocial well-being and the realtionship between divorce and children's academic performance. Journal of Marriage and Family, 72(4), 933-946. doi: 10.1111/j.1741-3737.2010.00740.x

Sandler, I. N., Tein, J., Mehta, P., Wolchik, S., y Ayers, T., (2000). Coping Efficacy and Psychological Problems of Children of Divorce. Child Development, 71(4), 10991118 .

Sweeney, R. B., y Bracken, B. A. (2000). Influence of family structure on children's selfconcept development. Canadian Journal of School Psychology, 16(1), 39-52.

Tillman, K.H. (2007). Family structure pathways and academic disadvantage among adolescents in stepfamilies. Sociological Inquiry, 77, 383 - 424.

Van Amerigen, M., Mancini, C., y Farvolden, P. (2003). The impact of anxiety disorders on educational achievement. Journal of Anxiety Disorders, 17, 561-571. 